\title{
INTERPROVINCIAL RECOVERIES \\ OF MOUNTAIN BLUEBIRDS AND TREE SWALLOWS, AND A CALIFORNIA RECOVERY OF A TREE SWALLOW
}

DONALD J. STILES, 20 Lake Wapta Rise SE, Calgary, AB T2J 2M9.

Often, migrating Mountain Bluebirds and Tree Swallows return to near their nesting sites in subsequent seasons. Calgary area banders have found that a bird banded as an adult may even return to the same nest box it used the previous year, or usually at least within five $\mathrm{km}$. They have found that birds banded as nestlings disperse and are more likely found further away in subsequent years than those banded as adults, but often within twenty four kilometres of their natal site. Occasionally, a bird will get off track and return to a different province. A few birds which have done this are described below.

\section{Mountain Bluebirds:}

8051-54109, a Mountain Bluebird was banded as a nestling in 1993 by Jim Speer, near Shellmouth, MB, just east of the Sask. border west of Riding Mountain National Park, and recovered as an incubating female in a nest box east of Priddis, $A B$ by Andrew Holder, a subpermittee of Don Stiles, on June 13, 1996. This is the first band recovery from Manitoba to Alberta. The recovery location is $890 \mathrm{~km}$. straight west of the banding location.

This prompted an examination of the recoveries of Mountain Bluebirds to determine whether there were any other "interprovincial" recoveries. Table 1 and Figure 1 show four other "interprovincial" recoveries recorded from 1970 to 1994 found in data supplied by Canadian Wildlife Service. One moved from SK to $M B$, one from $S K$ to $A B$, and two moved from $A B$ to $S K$.

Distances varied from $155 \mathrm{~km}$ (near Provost, $A B$ to east of North Battleford, $\mathrm{SK}$ ) to $680 \mathrm{~km}$ (near Viking, $A B$ to east of Melville, SK). Three were recovered alive in nest boxes and two were found dead. All of these birds except the first one in Table 1 were banded as nestlings, then travelled south on migration and returned to another province one or more years later. The exception, 1091-36438 was banded as an adult female by Lorne Scott on May 24, 1973 near Grenfell, SK, and was captured in a nest box near Souris, MB on June 21 , 1973 , a surprising distance of $200 \mathrm{~km}$ from where banded.

A Mountain Bluebird, 991-05960, recovered in Vancouver, BC, (Lat-Long 49 $10^{\prime}, 1230^{\prime}$ ) on Aug. 10, 1987, is not listed in Table 1. A phone call to the band recoverer, revealed that he was a long distance trucker, and the banded bird had been found dead by his son on the flatbed of his truck while it was being cleaned. He had recently hauled a load of prefabricated log houses from Cochrane, $A B$ to Vancouver. This bird was banded by Marijke Jalink, a subpermittee of Don Stiles, on July 13 , 1987 near Millarville, AB (Lat-Long 50 $\left.40^{\prime}, 11420^{\prime}\right)$. After it fledged, it is speculated that the bird flew about $50 \mathrm{~km}$ north 


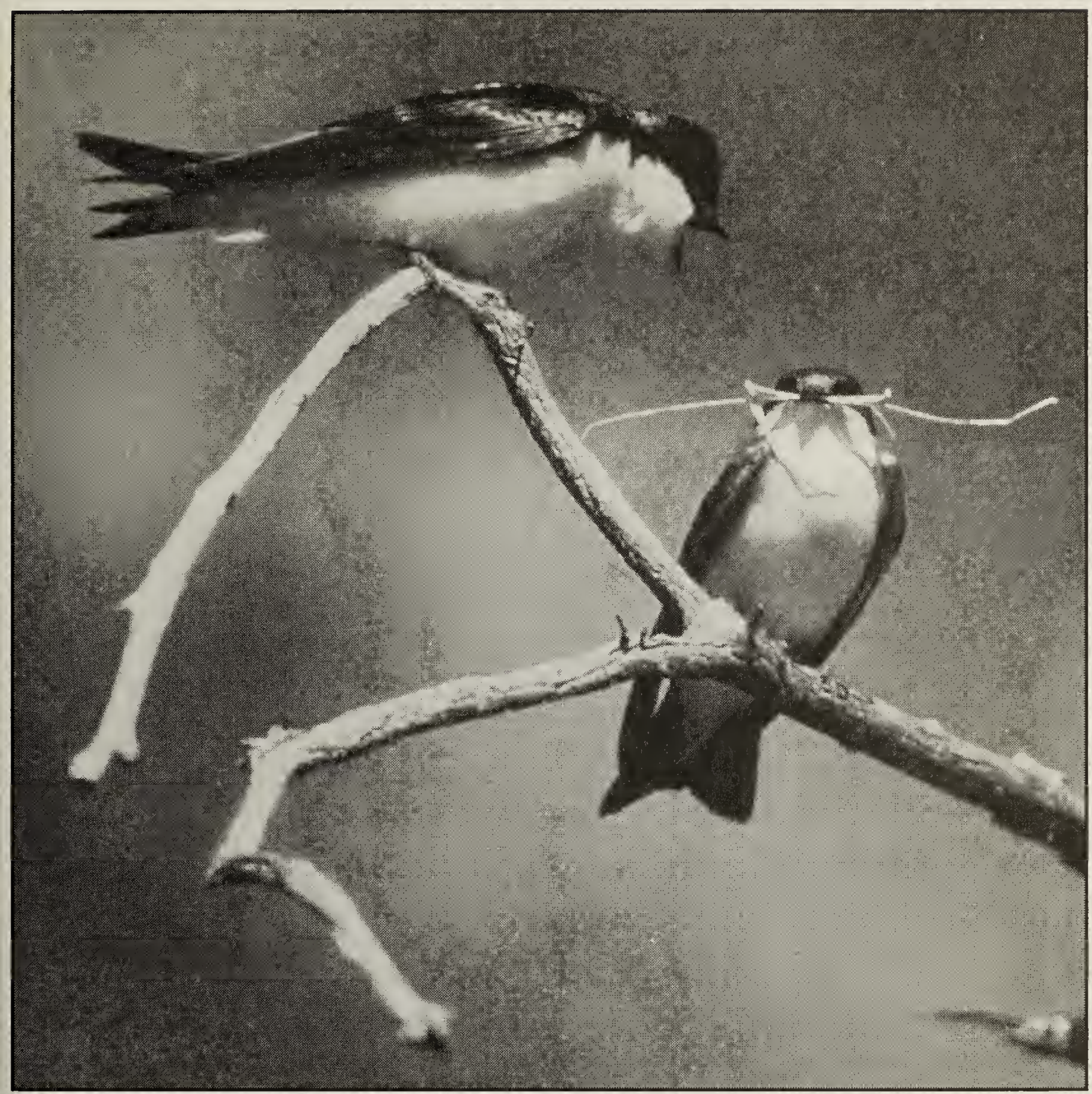

to Cochrane or vicinity where it was hit by the truck. It then travelled to Vancouver on the truck bed and was recovered on August 10.

\section{Tree Swallows:}

Only two Tree Swallow interprovincial recoveries were found in the Canadian Wildlife Service data up to 1992. One banded in Saskatchewan was recovered in Manitoba, and one vice versa. One of these, 520-49136, was banded as an adult in 1958 and recovered in 1960 , making this bird at least three years old. The second, 880-41966, was banded as a nestling by Mary Houston on July 7, 1977 near Pike Lake, SK, and recovered near Altamont, MB after hitting a power line in August 1977. Presumably this bird had already begun its migration.

\section{A California Tree Swallow Recovery:}

121-85793, a Tree Swallow banded as a nestling by George Loades, a subpermittee of Don Stiles, on July 8, 1995 on his Jumping Pound bluebird trail southwest of Cochrane, $A B$, was recovered alive in a mist net at the Modoc National Wildlife Refuge in northeastern California on April 12, 1997. (See Table 1 and Figure 1) This is the 


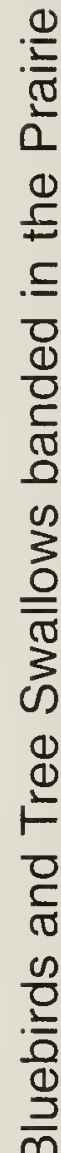

\begin{tabular}{|c|c|c|c|c|c|c|c|c|c|c|c|c|c|c|c|c|}
\hline & & 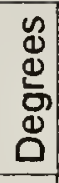 & & $\begin{array}{l}\omega \\
\omega \\
\stackrel{2}{0} \\
\stackrel{0}{2}\end{array}$ & $\begin{array}{l}3 \\
0 \\
N \\
N\end{array}$ & $\begin{array}{l}\omega \\
\omega \\
0 \\
\end{array}$ & $\begin{array}{l}\text { U } \\
\$ 0 \\
6\end{array}$ & $\begin{array}{l}3 \\
0 \\
N\end{array}$ & 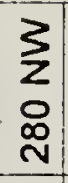 & $\begin{array}{l}山 \\
\sim \\
m \\
=\end{array}$ & $\begin{array}{l}3 \\
\text { is } \\
\infty \\
0 \\
\end{array}$ & & & & & \\
\hline & & $\begin{array}{l} \\
0 \\
\frac{1}{\pi} \\
\frac{\pi}{50} \\
0 \\
0\end{array}$ & $\begin{array}{l}\bar{E} \\
\underline{\Sigma}\end{array}$ & 유 & i & $\begin{array}{l}1 \\
0 \\
0\end{array}$ & 足 & 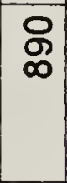 & 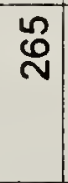 & Oे & $\stackrel{n}{=}$ & & & & & \\
\hline & & 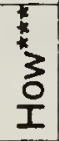 & & m & m & 0 & 0 & $m$ & ले & 灾 & D & & & & & \\
\hline & & $\begin{array}{l}0 \\
\\
\end{array}$ & & - & $N$ & 17 & $\theta$ & $m$ & $m$ & 0 & $N$ & & & & & \\
\hline & & $\begin{array}{l}\frac{*}{*} \\
\frac{0}{\pi} \\
\frac{0}{\pi}\end{array}$ & & $\begin{array}{l}\bar{N} \\
\text { O̊ } \\
\text { N }\end{array}$ & $\frac{m}{\frac{m}{5}}$ & $\mid \begin{array}{l}\infty \\
\frac{1}{2} \\
0 \\
0 \\
\infty \\
\infty\end{array}$ & 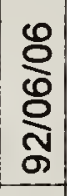 & 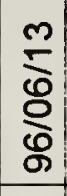 & 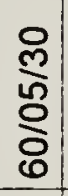 & 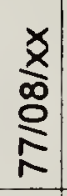 & $\frac{N}{\frac{N}{\sigma}}$ & & \multirow{3}{*}{\multicolumn{4}{|c|}{ 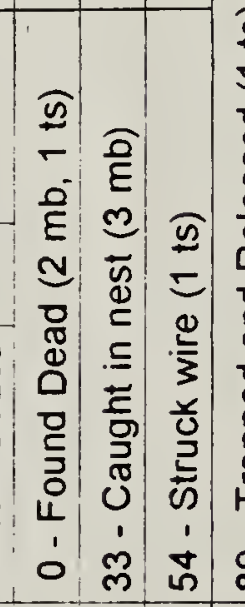 }} \\
\hline & 离 & 它 & & $\sum^{\mathscr{D}}$ & $\stackrel{\mathscr{Q}}{<}$ & $\frac{x}{\omega}$ & $\frac{x}{\omega}$ & 罗 & 文 & $\sum_{\Sigma}^{\mathbf{m}}$ & 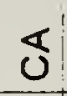 & & & & & \\
\hline 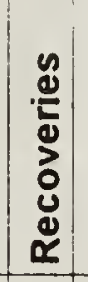 & $\begin{array}{l}\mathbf{U} \\
0 \\
\mathbf{d} \\
\mathbf{U} \\
\mathbf{0} \\
\mathbf{U} \\
\mathbf{3}\end{array}$ & 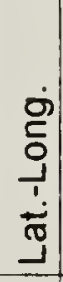 & & $\begin{array}{l}\text { 오 } \\
8 \\
8 \\
0 \\
0 \\
\dot{8} \\
\text { g }\end{array}$ & 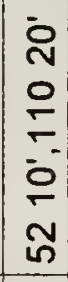 & $\begin{array}{c}0 \\
y \\
2 \\
0 \\
0 \\
0 \\
8 \\
0 \\
0\end{array}$ & $\begin{array}{c}0 \\
0 \\
\infty \\
0 \\
0 \\
0 \\
\gamma \\
\sim \\
\text { N }\end{array}$ & $\begin{array}{l}0 \\
0 \\
5 \\
5 \\
0 \\
6 \\
0 \\
0\end{array}$ & 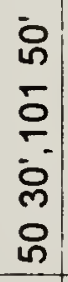 & $\begin{array}{l}0 \\
0 \\
\infty \\
o \\
- \\
i \\
o \\
\text { o }\end{array}$ & 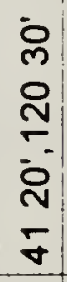 & $\begin{array}{l}0 \\
0 \\
0 \\
0 \\
3 \\
0 \\
1 \\
*\end{array}$ & & & & \\
\hline
\end{tabular}

ำ

ફ

정

ฐ

$\sum$

吹

क क

(1)

ठ논

(1)

承

$\bar{\sigma}$

응

$\risingdotseq \circlearrowleft$

은 0

은음

咅

$\begin{array}{ll}1 & 0 \\ \div & 0 \\ 0 & 0 \\ \frac{0}{0} & 0\end{array}$

\begin{tabular}{|c|c|c|c|c|c|c|c|c|c|c|c|c|c|c|c|c|c|c|}
\hline & & $\begin{array}{l}\frac{1}{\pi} \\
0\end{array}$ & $\begin{array}{l}\text { 足 } \\
\text { 足 } \\
\text { 岃 }\end{array}$ & 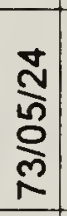 & 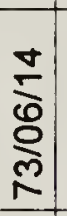 & 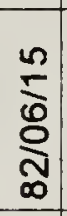 & 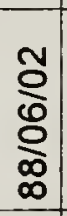 & 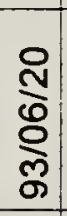 & $\stackrel{n}{3}$ & 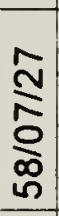 & $\begin{array}{l}\infty \\
\stackrel{0}{N} \\
\stackrel{2}{\Omega} \\
\end{array}$ & 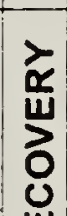 & $\begin{array}{l}\infty \\
0 \\
5 \\
0 \\
0 \\
0\end{array}$ & & & & 믕 & \\
\hline & & ن & 畐 & 品 & 品 & $\stackrel{\infty}{4}$ & $\stackrel{\infty}{\mathbb{Q}}$ & $\underline{\mathbf{m}}$ & لِ & $\underline{\mathbf{m}}$ & $\begin{array}{r}x \\
\omega\end{array}$ & $\underset{\underline{\underline{x}}}{\mathbf{u}}$ & 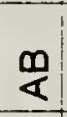 & & & & $\frac{2}{\Delta}$ & \\
\hline 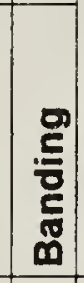 & 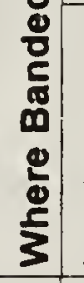 & 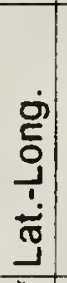 & $\begin{array}{l}\frac{2}{\alpha} \\
\frac{5}{2} \\
\frac{2}{2} \\
\frac{0}{2} \\
\frac{1}{5}\end{array}$ & 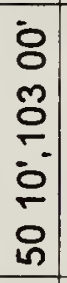 & 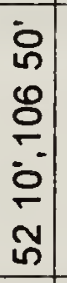 & 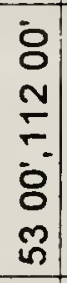 & 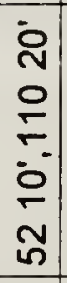 & $\begin{array}{c}0 \\
\text { ' } \\
0 \\
0 \\
0 \\
0 \\
0 \\
0\end{array}$ & 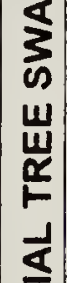 & $\begin{array}{c}0 \\
0 \\
\infty \\
o \\
0 \\
0 \\
0 \\
0\end{array}$ & $\begin{array}{c}0 \\
y \\
6 \\
0 \\
0 \\
6 \\
5 \\
5\end{array}$ & 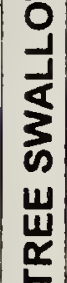 & $\begin{array}{c}0 \\
0 \\
\pm \\
\mp \\
8 \\
8 \\
5\end{array}$ & & & & 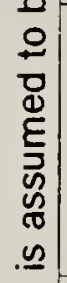 & \\
\hline & & $\begin{array}{l}* \\
0 \\
0 \\
\mathbb{\alpha}\end{array}$ & $\stackrel{\bar{J}}{ٍ}$ & $u$ & - & - & - & 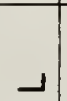 & $\stackrel{\bar{U}}{\mathbf{z}}$ & $\begin{array}{l}\overrightarrow{1} \\
\\
\end{array}$ & ـ & $\frac{\Phi}{z}$ & 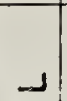 & & & $\sum_{3}$ & 宅 & \\
\hline & & $\begin{array}{l}0 \\
Z \\
\text { D } \\
\mathbb{D} \\
\infty\end{array}$ & $\begin{array}{l}0 \\
\alpha \\
a \\
\alpha \\
w \\
\underline{\underline{\alpha}} \\
\underline{z}\end{array}$ & 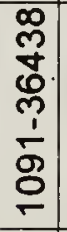 & $\begin{array}{l}0 \\
0 \\
0 \\
+ \\
1 \\
1 \\
0 \\
\end{array}$ & $\begin{array}{l}\hat{m} \\
0 \\
\\
\frac{1}{9} \\
\infty\end{array}$ & 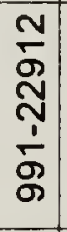 & $\begin{array}{l}0 \\
0 \\
\overline{4} \\
1 \\
50 \\
0 \\
0\end{array}$ & $\begin{array}{l}0 \\
\alpha \\
0 \\
\alpha \\
w \\
\underline{\underline{w}} \\
\underline{z}\end{array}$ & 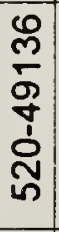 & 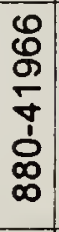 & 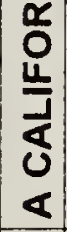 & $\begin{array}{l}0 \\
o \\
N \\
0 \\
1 \\
N \\
\end{array}$ & $\begin{array}{l}2 \\
\frac{2}{0} \\
0 \\
0 \\
0 \\
0 \\
5\end{array}$ & $\begin{array}{l}\frac{\mathscr{U}}{\widetilde{N}} \\
\frac{\mathrm{D}}{\mathcal{D}}\end{array}$ & 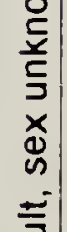 & $\begin{array}{l}\overline{0} \\
0 \\
\pi \\
0 \\
0 \\
0 \\
0 \\
5\end{array}$ & $\sum_{0}$ \\
\hline & & 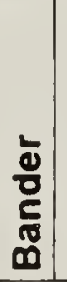 & & $\begin{array}{l}\tilde{z} \\
\delta \\
\tilde{n} \\
\dot{u} \\
\ddot{\alpha}\end{array}$ & 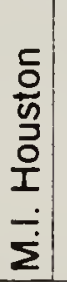 & $\begin{array}{l}\frac{\vec{\omega}}{C} \\
i \bar{u} \\
\dot{0} \\
\dot{j}\end{array}$ & 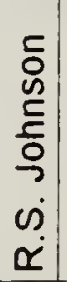 & $\begin{array}{l}0 \\
\Phi \\
0 \\
0 \\
;\end{array}$ & & $\begin{array}{l}\frac{0}{\lambda} \\
\stackrel{\sigma}{\sigma} \\
\dot{\alpha} \\
\alpha\end{array}$ & $\begin{array}{l}\frac{5}{0} \\
\text { ș } \\
\text { 우 } \\
\dot{\bar{\Sigma}}\end{array}$ & & $\begin{array}{l}\mathscr{d} \\
\stackrel{\bar{\varpi}}{ } \\
\dot{0} \\
\dot{0}\end{array}$ & 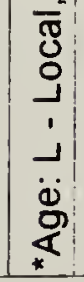 & $\begin{array}{l}\frac{5}{3} \\
\frac{7}{4} \\
4 \\
4\end{array}$ & $\begin{array}{l}0 \\
8 \\
1 \\
? \\
1 \\
0\end{array}$ & 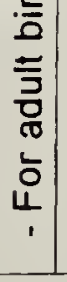 & 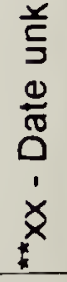 \\
\hline
\end{tabular}




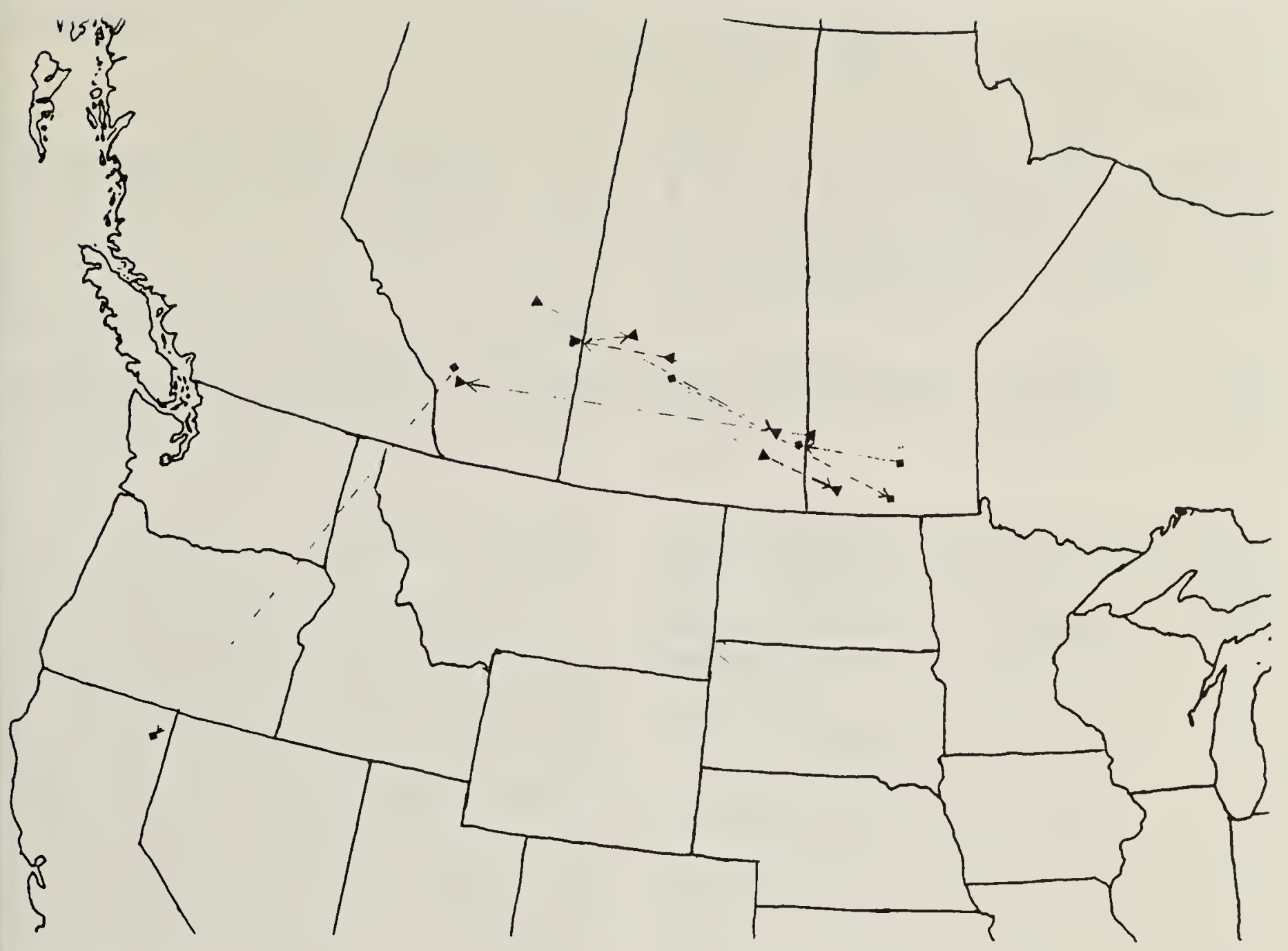

Figure 1. Interprovincial Recoveries of Mountain Bluebirds and Tree Swallows, and a California Tree Swallow Recovery. - Mountian Bluebirds. $\mathbf{\square}$ - Tree Swallows

first Tree Swallow banded in Alberta that has travelled southwest on migration. All 16 previously banded in Alberta have been recovered to the southeast ${ }^{1,2}$. One factor in this may be that this bird was banded in the foothills southwest of Calgary, one of the more westerly locations in Alberta where banding is done.

Ronnie L. Ryno, Assistant Manager at the wildlife refuge reports that they capture Tree Swallows in April and early May in mist nets, most of which later nest nearby in nest boxes. Also of interest in his letter: "during March and April we often get cold spells when the temperatures will drop into the low teens $\left({ }^{\circ} \mathrm{F}\right)$, this usually results in dozens or even hundreds of dead Tree Swallows around their roost sites, especially if the cold weather lasts a few days and/or is combined with snow." This is another indi- cation that weather plays a big factor in bird migration. Another Tree Swallow recovered in Nebraska, at much the same latitude as the above, died due to severe weather ${ }^{2}$.

1. Stiles, D.J. and M.I. Houston. 1994. Distant Travels of Tree Swallows of the Prairie Provinces, 1968-1992. Blue Jay 52:206-212.

2. Stiles, D.J. 1997. Recoveries of Mountain Bluebirds south of $49^{\circ}$ Latitude, and a recent Tree Swallow. Blue Jay 55:48-52. 